\title{
An Analysis of Fuzzy and Spatial Methods for Edge Detection
}

\author{
Pushpa Mamoria \\ Department of Computer Science, Babasaheb Bhimrao Ambedkar University, Lucknow, India \\ Email:p.mat76@gmail.com \\ Deepa Raj \\ Department of Computer Science, Babasaheb Bhimrao Ambedkar University, Lucknow, India \\ Email: Deepa_raj200@yahoo.co.in
}

\begin{abstract}
An image segmentation is an area in which image is subdivided into sub-regions for extracting characteristics of images which will help to analysis in various applications. For getting accuracy sharp changes of intensity is an important issue which is known as edge detection. In this paper various spatial edge detection methods and fuzzy based edge detection method has described and spatial edge detection methods and fuzzy if-then-else are compared to know which method will be more suitable to find edges for the enhancement of images.
\end{abstract}

Index Terms - Image segmentation, Edge, Threshold, Fuzzy method.

\section{INTRODUCTION}

A method in which various input image properties is obtained as an output known as transition. Transition state of the input image to output image lay in the segmentation.

An Image segmentation splits an image into its subregions to collect the details which are found in a subregion of the image. These details are helpful to analyzing images for the enhancement of images. The accuracy of enhancement is based on accurate segmentation method. For this reason, more care should be taken to improve images with the help of accurate segmentation. It is helpful to control the environment in many applications like in military to detect objects, industrial inspection applications etc. An image segmentation method has two types of categories to achieve accuracy. The first category is describing a partition of images based on Sharpe changes in intensity known as edge and the second category is based on a set of predefined criteria known as Thresholding, region growing and region splitting and merging [1] [2]. With the help of thresholding edge, detection can be possible to overcome the criteria of the noisy condition. In this method first order derivative of Gaussian filter used for convolving the images [3]. Edges found by canny can also create some false edges. The structure of image can also find with the help of method known as USAN, which is helpful in edge detection [4]. Edges may also find with the help of zero crossing [5]. For measurement of the degree of fuzziness, entropy has used, which produced 1-pixel wide edges [6]. Image segmentation is a difficult task to make images meaningful. By using canny method image segmentation may give many false edges to increase the complexity of image characteristics. Instead of Canny method, a two steps Chan These method is helpful to detect best edges [7]. All the classical spatial methods like Canny edge detector, Sobel method, Prewitt edge detection, and Laplacian of Gaussian are not been able to detect correct and smooth edges in images. Latterly a new method intuitionistic fuzzy set (IFS) theory was proposed to detect correct and smooth edges. The intuitionistic fuzzy method used the concept of entropy in various clustering algorithm [9].

The remaining paper is divided as follows. Section II is describing various classical edge detection techniques available in the literature. Section III is describing fuzzy domain edge detection techniques. Section IV is based on experimental study and results. And in the last section $\mathrm{V}$ conclusions are drawn.

\section{Spatial Domain Edge Detection Techniques}

Spatial filtering is used in the Edge detection to used break off between gray levels. For the edge detection in images first order derivative and second order derivatives are used. First order derivative are worked out by using gradient and second order derivative are found by the Laplacian.

\section{A. Gradient operator}

The gradient of an image $f(x, y)$ at a location $(x, y)$ is defined as the vector:

$$
\nabla f=\left[\frac{G_{x}}{G_{y}}\right]=\left[\frac{\partial t / \partial x}{\partial t / \partial y}\right]
$$

Magnitude of above vector:

$$
\nabla f=\operatorname{mag}(\nabla f)=\sqrt[2]{G_{x}^{2}}+\sqrt[2]{G_{y}^{2}}
$$

The direction of the gradient vector: 


$$
\alpha(x, y)=\tan ^{-1}\left(\frac{G x}{G y}\right)
$$

Different types of edge detection methods are based on three categorizations:

\section{B. Classical edge detection methods:}

\section{i. $\quad$ Sobel Mask}

Mask used by this method is:

\begin{tabular}{|c|l|l|}
\hline-1 & -2 & -1 \\
\hline 0 & 0 & 0 \\
\hline 1 & 2 & 1 \\
\hline
\end{tabular}$\quad$\begin{tabular}{|l|l|l|}
\hline-1 & 0 & 1 \\
\hline-2 & 0 & 2 \\
\hline-1 & 0 & 1 \\
\hline
\end{tabular}

Fig.1. Sobel Mask

Using the below equations Sobel mask is used for edge detection.

$$
\begin{aligned}
& G_{x}=\left(Z_{7}+2 Z_{8}+Z_{9}\right)-\left(Z_{1}+2 Z_{2}+Z_{3}\right) \\
& G_{y}=\left(Z_{3}+2 Z_{6}+Z_{9}\right)-\left(Z_{1}+2 Z_{4}+Z_{7}\right)
\end{aligned}
$$

\section{ii. $\quad$ Prewitt Mask}

Mask used by this method is:

\begin{tabular}{|l|l|l|}
\hline-1 & -1 & -1 \\
\hline 0 & 0 & 0 \\
\hline 1 & 1 & 1 \\
\hline
\end{tabular}

\begin{tabular}{|l|l|l|}
\hline-1 & 0 & 1 \\
\hline-1 & 0 & 1 \\
\hline-1 & 0 & 1 \\
\hline
\end{tabular}

Fig.2. Prewitt Mask

Using the below equations Prewitt mask is used for edge detection.

$$
\begin{aligned}
& G_{x}=\left(Z_{7}+Z_{8}+Z_{9}\right)-\left(Z_{1}+Z_{2}+Z_{3}\right) \\
& G_{y}=\left(Z_{3}+Z_{6}+Z_{9}\right)-\left(Z_{1}+Z_{4}+Z_{7}\right)
\end{aligned}
$$

\section{iii. Robert Mask}

Mask used by this method is:

\begin{tabular}{|c|l|}
\hline-1 & 0 \\
\hline 0 & 1 \\
\hline
\end{tabular}

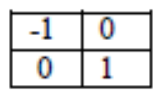

Fig.3. Robert mask

Using the below equations Robert mask is used for edge detection.

$$
\begin{aligned}
& G_{x}=\left(Z_{9}-Z_{5}\right) \\
& G_{y}=\left(Z_{8}-Z_{6}\right)
\end{aligned}
$$

\section{First order edge detection method}

i. Canny edge detection
- It uses smoothing as a process to remove noise.

- Finding the gradient of images which has a large magnitude.

- $\quad$ By using double thresholding.

\section{Second, order edge detection method}

\section{i. Laplacian}

The Laplacian of a 2-D function $f(x, y)$ is a secondorder derivative. The Laplacian is merged with smoothing to find edges via zero-crossing.
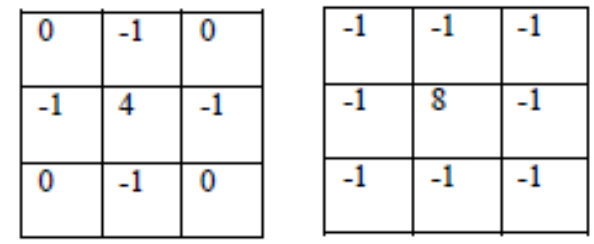

Fig.4. Laplacian mask

In Laplacian filtering enhanced image can be found by:

$$
F^{\prime}(x, y)=F(x, y)+C \nabla F(x, y)
$$

\section{ii. Laplacian of a Gaussian (LOG)}

Laplacian of a Gaussian sometimes is called the Mexican hat function. Gaussian function is used to smooth the image and Laplacian operator is used to constitute the location of edges by zero findings. Here, Figure- 5 is a $5 \times 5$ mask to approximate the shape of Maxican hat function.

\begin{tabular}{|l|l|l|l|l|}
\hline 0 & 0 & -1 & 0 & 0 \\
\hline 0 & -1 & -2 & -1 & 0 \\
\hline-1 & -2 & 16 & -2 & -1 \\
\hline 0 & -1 & -2 & -1 & 0 \\
\hline 0 & 0 & -1 & 0 & 0 \\
\hline
\end{tabular}

Fig.5. Laplacian of Gaussian Filter

\section{FUZZY DOMAIN EDGE DETECTION TECHNIQUES}

In many applications, edge detection becomes very important due to the criticality of use of images like in medical images diagnosis of disease is very crucial. Due to this reason, images must be free of poor contrast, vagueness, blurred or broken edges. That's why because of the above reasons fuzzy method is suitable to take into account the unclearness and equivocalness present in the image [17]. Several Fuzzy edge detection methods are following: 


\section{A. Fuzzy Sobel edge detector [13]}

In this method the image is divided into two regions:

\section{i. Fuzzy edge region}

In this method, if pixels have a high difference in the gray level with their neighborhood region then the pixels are separated by the fuzzy edge region.

\section{ii. Fuzzy smooth region}

In this method, if pixels have less difference of gray level with their neighborhood region then the pixels are separated by the fuzzy smooth region.

By using fuzzy reasoning the modified fuzzy edge detector is generalized by using following fuzzy rules:

$$
\begin{gathered}
\mathrm{R}(\mathrm{x}, \mathrm{y})=255, \text { if } \mathrm{G}(\mathrm{x}, \mathrm{y}) \geq \mathrm{HT} \\
=0, \text { if } \mathrm{G}(\mathrm{x}, \mathrm{y}) \leq \mathrm{LT} \\
=\mathrm{G}(\mathrm{x}, \mathrm{y}) \cdot \max \left(\mu_{S F R}(\mathrm{x}, \mathrm{y}), \mu_{E F R}(\mathrm{x}, \mathrm{y})\right) \\
\text { Otherwise }
\end{gathered}
$$

Where $\mu_{S E R}$ and $\mu_{E F R}$ are the membership functions of the image smooth and edge regions.

$\mathrm{G}(\mathrm{x}, \mathrm{y})$ is the gradient value using Sobel operator.

$\mathrm{R}(\mathrm{x}, \mathrm{y})$ is the resultant pixel at location $(\mathrm{x}, \mathrm{y})$.

\section{B. Entropy-Based Fuzzy Edge Detection [11]}

As per information theory, defined formula of entropy is given as:

$$
\begin{gathered}
\mathrm{H}(\mathrm{t} 1, \mathrm{t} 2)=-P_{\text {smooth }} \log _{2}\left(P_{\text {smooth }}\right)- \\
P_{\text {edge }} \log _{2}\left(P_{\text {edge }}\right)
\end{gathered}
$$

Where $P_{\text {edge }}$ and $P_{\text {smooth }}$ are

Probability distributions.

$$
\begin{gathered}
P_{\text {edge }}=\sum_{k} h g_{k} \cdot \mu_{\text {edge }}(\mathrm{k}) \\
P_{\text {smooth }}=\sum_{k} h g_{k} \cdot \mu_{\text {smooth }}(\mathrm{k})
\end{gathered}
$$

Here $P_{\text {smooth }}$ and $P_{\text {edge }}$ are weight area on the gradient histogram and when membership function $\mu_{\text {smooth }}$ and $\mu_{\text {edge }}$ are weights.

In edge detection, the best parameter values are compact edge representation of images that's why minimum entropy $\mathrm{H}\left(t_{1}, t_{2}\right)$ parameters are selected.

The necessary assumptions for minimum and maximum entropy are:

$$
\begin{gathered}
\partial \mathrm{H}\left(t_{1}, t_{2}\right) / \partial t_{1}=0=-\left(\partial P_{\text {smooth }} / \partial t_{1} \mathrm{X} \log \left(P_{\text {smooth }} /\right.\right. \\
\left.1-\left(P_{\text {smooth }}\right)\right)
\end{gathered}
$$

$$
\begin{gathered}
\partial \mathrm{H}\left(t_{1}, t_{2}\right) / \partial t_{2}=0=-\left(\partial P_{\text {smooth }} / \partial t_{2} \mathrm{X} \log \left(P_{\text {smooth }}\right.\right. \\
\left.\left./ 1-P_{\text {smooth }}\right)\right)
\end{gathered}
$$

The entropy will be minimum,

$$
\text { When } P_{\text {smooth }}=P_{\text {edge }}=1 / 2
$$

The best set of parameter $\left(\widetilde{t_{1}}, \widetilde{t_{2}}\right)$ will satisfy the following condition:

$$
\mathrm{H}\left(\widetilde{t_{1}}, \widetilde{t_{2}}\right)=\min _{r=0,1,2 \ldots R}\left(H\left(t_{1}(\mathrm{r}), t_{2}(\mathrm{r})\right)\right)
$$

The edge image is calculated as

$$
\text { Edge }- \text { image }(x, y)=1
$$

\section{Fuzzy template based edge detector}

Two types of fuzzy template based edge detector method are available.

In first method, fuzzy edge templates are designed and these templates are convolved with the image [12].

For finding the existence of edge:

$$
\mathrm{H}(\mathrm{x}, \mathrm{y})=\max _{T=1, \text { to } n}\left(H_{T}(x, y)\right)
$$

Where $n=$ number of templates.

The new images are found with the support of threshold, the value which is below the threshold are set as 0 and above the threshold are set as 1 .

In second method fuzzy divergence used in between image window and a set of 16 fuzzy templates [8].

\section{Fuzzy If-then Rules based Edge Detection}

This fuzzy technique is based on rule-based fuzzy logic. The fuzzy rule-based concept has been taken from Fuzzy set theory [14] [15] because of its simplicity and effectiveness. It is an inference form of uncertain knowledge to handle and analyze information in an effective manner. It could be combined different filters along with fuzzy if-then rules to detect edges for enhancement of images [16]. For edge detection fuzzy logic algorithm using following rules [1]:

(1) If a pixel belongs to the same region, then make it brighter; else make it darker, where values related to brighter and darker are fuzzy sets.

(2) A 3x3 pixel neighborhood and corresponding intensity differences between the center pixels and its neighbors are shown below.

\begin{tabular}{|l|l|l|}
\hline$z_{1}$ & $z_{2}$ & $z_{3}$ \\
\hline$z_{4}$ & $z_{5}$ & $z_{6}$ \\
\hline$z_{7}$ & $z_{8}$ & $z_{9}$ \\
\hline
\end{tabular}

\begin{tabular}{|c|c|c|}
\hline$d_{1}$ & $d_{2}$ & $d_{3}$ \\
\hline$d_{5}$ & 0 & $d_{6}$ \\
\hline$d_{7}$ & $d_{8}$ & $d_{9}$ \\
\hline
\end{tabular}

Fig.6. $3 \times 3$ mask

The followings are the if-then-else rules based on fuzzy values.

IF $d_{2}$ is zero AND $d_{6}$ is zero THEN $z_{5}$ is white IF $d_{6}$ is zero AND $d_{8}$ is zero THEN $z_{5}$ is white IF $d_{8}$ is zero $A N D d_{4}$ is zero THEN $z_{5}$ is white IF $d_{4}$ is zero AND $d_{2}$ is zero THEN $z_{5}$ is white ELSE $z_{5}$ is black 
Membership functions $\mathrm{ZE}, \mathrm{BL}$, and $\mathrm{WH}$ is used for the fuzzy sets zero, black, and white.

\section{EXPERIMENTAL STUDY AND RESULTS}

In this study, several images have taken from internet and Matlab for the experiment of edge detection. Followings are the procedure of edge detection using fuzzy If-then rules:

Step-1: Apply the simple filters (convolution) to obtain the image gradients.

Step-2: Define the image gradients of input image with respect to $\mathrm{x}$-axis and $\mathrm{y}$-axis direction.

Step-3: Design a Fuzzy Inference System (FIS) with 2 inputs and 1 output.

Step-4: Set the Fuzzy-If Then Rules into FIS.

Step-5: Obtain the membership function of image gradients as Ix and Iy.

Step-6: Apply the Ix and Iy to above FIS for Evaluation.

Step-7: Store the result obtained from output of FIS.

This resultant would produce the edge of the image.

In this, we are comparing various classical method of edge detection with fuzzy if-then-else rule-based edge detection method. Based on performance evaluation parameters, we can compare various methods.

In the experiments, we have compared various methods of edge detection on the basis of two performance analysis parameters. One is PSNR and another is MSE, below every output image these values are written and we can compare on the basis of these values, which method is giving better results.

The mathematical formula of the PSNR is as follows:

$$
\operatorname{PSNR}=20 \log _{10}\left(\frac{255}{\sqrt{M S E}}\right)
$$

Where, MSE (Mean square error) is as follows:

$$
\text { MSE }=\frac{1}{m n} \sum_{0}^{m-1} \sum_{0}^{n-1}|| f(i, j)-g(I, j)||^{2}
$$

Where $\mathrm{f}=$ matrix data of the original image, $\mathrm{g}=$ matrix data of the degraded image, $m=$ no of rows of pixels of an image, $\mathrm{I}=$ index of that row, $\mathrm{n}=$ no of columns of pixels of an image, $\mathrm{j}=$ index of that column.

Higher the values of PSNR will show better results because only on the basis of visualization strongly we cannot reach the conclusion, which method is better.

Table 1. MSE values of different images

\begin{tabular}{|c|c|c|c|c|}
\hline & Image 1 & Image 2 & Image 3 & Image 4 \\
\hline SOBEL & 12698.83 & 14161.36 & 17974.31 & 17702.02 \\
\hline PREWITT & 12698.91 & 14161.36 & 17974.38 & 17702.12 \\
\hline ROBERT & 12699.58 & 14163.92 & 17974.16 & 17702.16 \\
\hline $\begin{array}{c}\text { CANNY } \\
\text { FUZZY } \\
\text { IF-THEN } \\
\text { ELSE }\end{array}$ & 12683.91 & 14161.25 & 17962.88 & 17693.80 \\
\hline
\end{tabular}

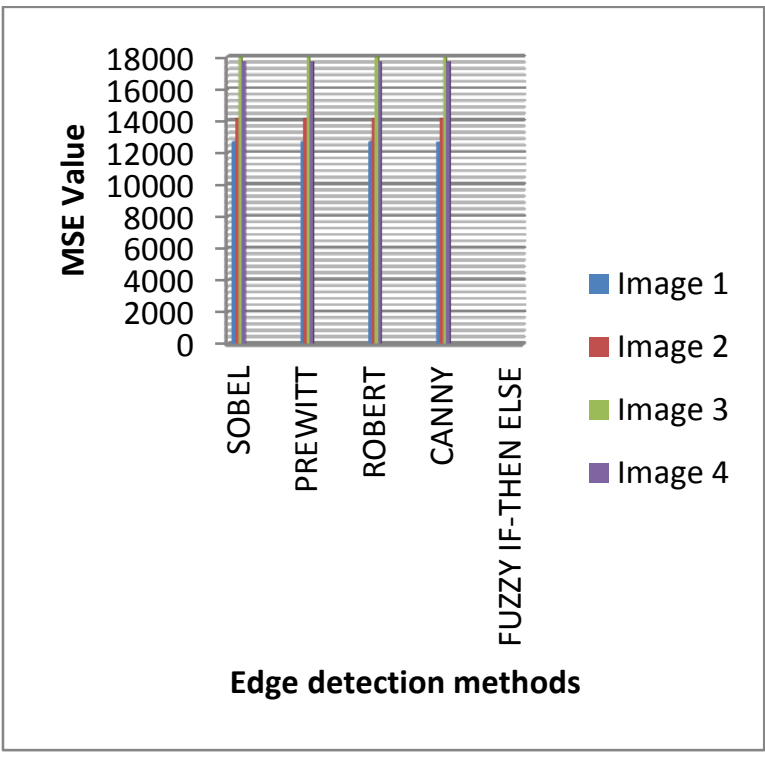

Fig.7. PSNR values of different images

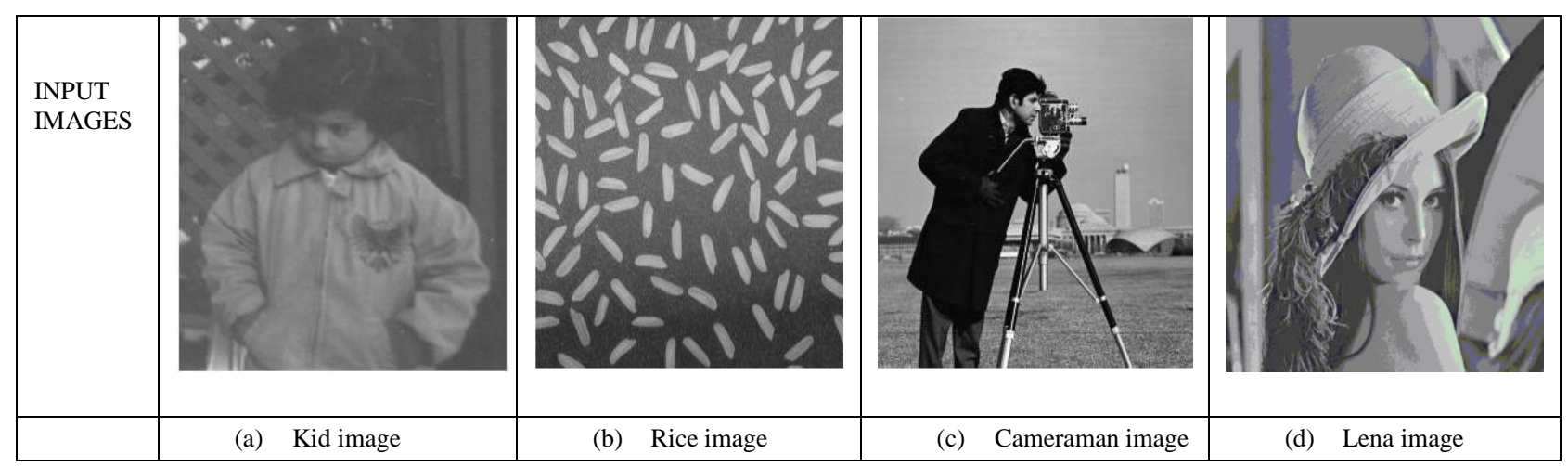




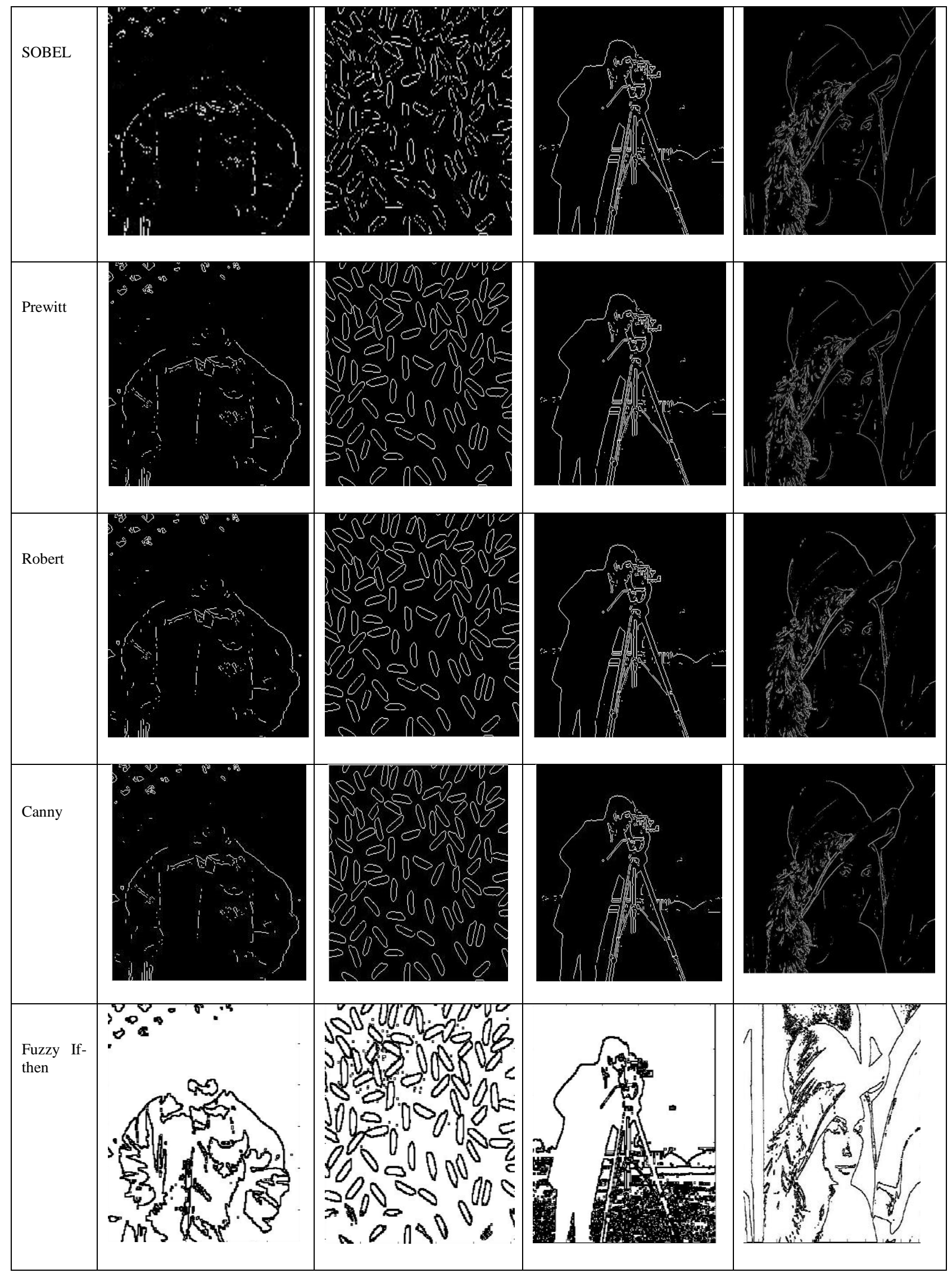

Fig.8. Results of various edge detection methods 
Table 2. PSNR values of different images.

\begin{tabular}{|l|l|l|l|l|}
\hline & Image 1 & Image 2 & Image 3 & Image 4 \\
\hline SOBEL & 7.40 & 6.65 & 5.62 & 11.71 \\
\hline PREWITT & 7.40 & 6.65 & 5.62 & 11.71 \\
\hline ROBERT & 7.40 & 6.65 & 5.62 & 11.71 \\
\hline CANNY & 7.40 & 6.65 & 5.62 & 11.71 \\
\hline $\begin{array}{l}\text { FUZZY IF- } \\
\text { THEN ELSE }\end{array}$ & 53.14 & 52.79 & 54.34 & 59.87 \\
\hline
\end{tabular}

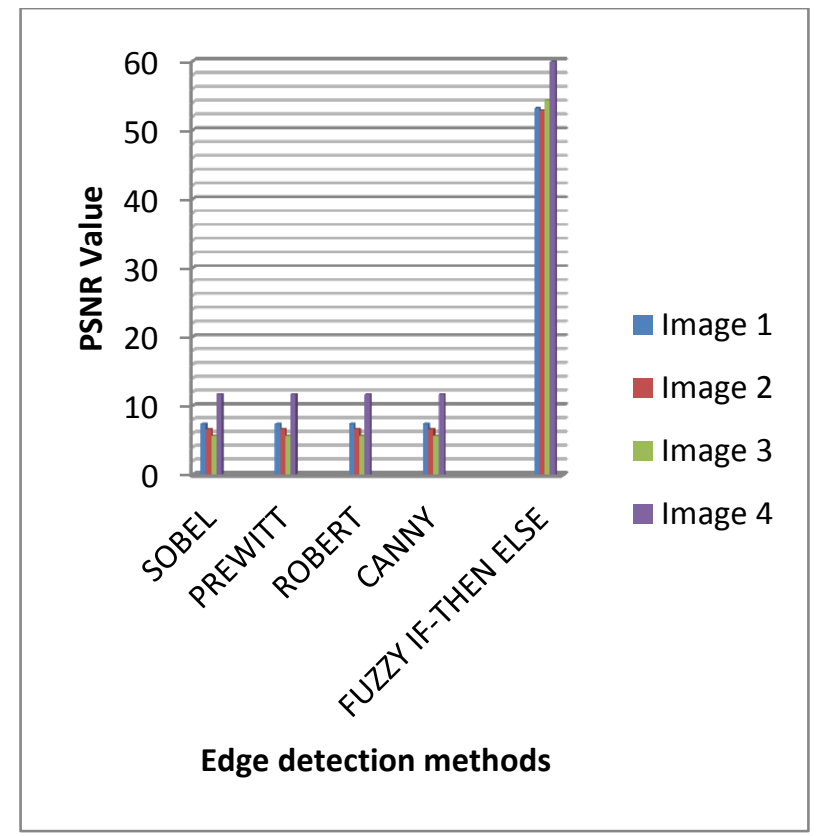

Fig.9. PSNR values of different images

\section{CONCLUSION}

In this paper, edge detection based on image segmentation of various spatial methods and fuzzy methods are discussed and compared the result of various spatial edge detection technique and fuzzy ifthen method. The experimental study is performed on various images collected from internet and Matlab. As per experimental results fuzzy method is giving better results as compared to spatial fuzzy methods like Sobel, Prewitt, Robert and Canny on the basis of visualization and with the help of quantitative values of performance parameters like PSNR, and MSE. The values of PSNR of the fuzzy method are higher as compared to other methods. Graphs of MSE values and PSNR values clearly showing better result of fuzzy if-then else method on the basis of their higher values of PSNR. However, fuzzy edge detection method is giving better results.

\section{REFERENCES}

[1] R. C. Gonzalez and R. E. Woods. "Digital Image Processing," 3rd ed. Prentice Hall, 2009.

[2] Jang, J.-S. R., C. T. Sun, and E. Mizutani, "Neuro-fuzzy and Soft Computing: A Computational Approach to Learning and Machine Intelligence," Prentice-Hall,
Upper Saddle River, NJ, 1997.

[3] J. F. Canny,"A computational approach to edge detection," IEEE Trans. On Pattern Analysis and Machine Intelligence. 8(6), 679/698, 1986.

[4] V. K. Madasu, S.Vasikarla, "Fuzzy edge detection in biometric systems," 36th Applied Imagery Pattern Recognition Workshop, IEEE, 2007.

[5] D. Marr, and E.C. Hildreth, "Theory of edge detection," Proc. Of the Royal Society of London, 187/217, 1980.

[6] M.Hanmandlu, J.See, and S.Vasikarla, "Fuzzy edge detector using entropy optimization",Proc. ITCC, 665/670, 2004.

[7] Y.-S. Chen, Y.-M. Chang, J.-C. Lin, "Comparing Intuitionistic Fuzzy Set Theory Method and Canny Algorithm for Edge Detection to Tongue Diagnosis in Traditional Chinese Medicine", Proc. Of the International conference of Information Application (ICCIA 2012), 2012.

[8] T. Chaira, and A.K. Ray, "A new measure using intuitionistic fuzzy set theory and its Application to edge detection," Applied Soft Computing, vol. 8-2, March, 2008, pp. 919- 927,doi:10.1016/j.asoc.2007.07.004, 2008.

[9] T. Chaira, "A novel Intuitionistic fuzzy-C means clustering algorithm and its application to medical images," Applied Soft Computing, vol.11-2, Mar. 2011, pp. 1711-1717, doi:10.1016/j.asoc.2010.05.005, 2011.

[10] P. R. Possa, S. A. Mahmoudi, NaimHarb, C. Valderrama, "A Multi-Resolution FPGA- Based Architecture for Real-Time Edge and Corner Detection", IEEE Transactions on Computers, January 2013.

[11] S. E. El-Khamy, I. Ghaleb, N. A. El-Yamany, "Fuzzy edge detection using minimum entropy", in Proceedings of 11th Mediterranean Electrotechnical Conference MELECON, Cairo, Egypt, 2002.

[12] Ho Kenneth, H.L., and Ohnishi, N., FEDGE-fuzzy edge detection by fuzzy categorization and classification of edges, in Fuzzy Logic in Artificial Intelligence, JCAI'95 Workshop, Selected Papers, pp. 182-196, 1995.

[13] Khamy, E.L. et al., Modified Sobel fuzzy edge detector, in Proceedings of 17th National Radio Science Conference (NRSC 2000), C32-1-9, Minui a, Egypt, 2000.

[14] L. A. ZADEH, "Fuzzy sets", Information and control 8, 338-353, 1965.

[15] K. Pal and R. A. King, "Image Enhancement using Fuzzy Set", Electronics Letters, Vol. 16, No. 10, May 1980.

[16] P. Mamoria, D. Raj, "An Analysis of Images Using Fuzzy Contrast Enhancement Techniques", $3^{\text {rd }} 2016$ International Conference on Computing for Sustainable Global Development, INDIACom-2016(IEEE Conference ID: 37465), BVICAM, New Delhi, India.

[17] S. K. Dubey, S. Panday, "Measurement of Usability of Office Application Using a Fuzzy Multi-Criteria Technique", IJITCS, MECS Publisher, Vol. 7, No 4, march 2015. 


\section{Authors' Profiles}

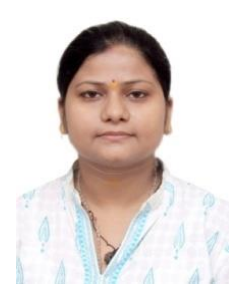

Pushpa Mamoria received her BE degree in Computer Science and Engineering from Shri G.S. Institute of Technology and Science, Indore (MP), INDIA and M. Tech. degree in Computer Science in from School of Computer Science, DAVV, Indore. She is currently pursuing her Ph.D. degree in the Department of Computer Science, Babasaheb Bhimrao Ambedkar University, Lucknow, India. Her major research interests include Digital Image Processing, Fuzzy Logic, Neural Network, Artificial Intelligence, cognitive science, wireless sensor networks.

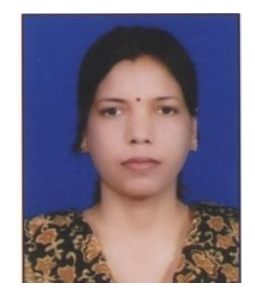

Dr. Deepa Raj, Working as an assistant professor in the Department of Computer Science Babasaheb Bhim Rao Ambedkar University. She did her Post Graduation from J.K Institute of applied physics and technology, Allahabad University and Ph.D. from Babasaheb Bhim Rao Ambedkar University Lucknow in the field of software engineering. Her field of interest is Software Engineering, Computer Graphics, and Image processing. She has attended lots of National and International conference and numbers of research papers published in her field.

How to cite this paper: Pushpa Mamoria, Deepa Raj,"An Analysis of Fuzzy and Spatial Methods for Edge Detection", International Journal of Information Engineering and Electronic Business(IJIEEB), Vol.8, No.6, pp.62-68, 2016. DOI: 10.5815/ijieeb.2016.06.08 